\title{
Percepção do cliente no perioperatório sobre o cuidado de enfermagem no centro cirúrgico
}

\author{
Perceptions of surgical patient during perioperative period concerning nursing care at the \\ surgical center
}
Percepciones del paciente quirúrgico durante el perioperatorio sobre el cuidado de enfermería en el centro quirúrgico

Itamara Barbosa Souza ${ }^{1 *}$, Hulda Alves de Araújo Tenório², Everaldo de Lima Gomes Junior ${ }^{1}$, Mário de Lucena Sarmento Neto ${ }^{1}$, Bruno Ribeiro de Almeida ${ }^{1}$, Emilly Souza Marques ${ }^{2}$.

\section{RESUMO}

Objetivo: Analisar a percepção do cliente do pré-operatório imediato e do transoperatório sobre os cuidados de enfermagem no centro cirúrgico. Métodos: Trata-se de uma pesquisa de campo, descritiva de abordagem qualitativa. Resultados: Os clientes referiram sentimentos de medo e ansiedade, porém receberam, em sua maioria, informações da equipe de enfermagem sendo ouvidos e acolhidos durante a experiência cirúrgica. Conclusão: A equipe de enfermagem desenvolveu uma assistência de qualidade baseada em princípios da humanização e da assistência integral, porém parece existir uma necessidade desses profissionais se adequarem a aspectos socioculturais dos clientes.

Palavras-chaves: Enfermagem Perioperatória, Cuidados de Enfermagem, Pacientes Internados.

\begin{abstract}
Objective: To analyze the client's perception of the immediate preoperative and the intraoperative period on the nursing care in the surgical center. Methods: This is a field research, descriptive of a qualitative approach. Results: The clients reported feelings of fear and anxiety, but received, in most, information from the nursing team being heard and welcomed during the surgical experience. Conclusion: The nursing team developed a quality care based on principles of humanization and integral care, but there seems to be a need for these professionals to adapt to the sociocultural aspects of clients.
\end{abstract}

Key words: Perioperative Nursing, Nursing Care, Inpatient Patients.

\section{RESUMEN}

Objetivo: Analizar la percepción del cliente del preoperatorio inmediato y del transoperatorio sobre los cuidados de enfermería en el centro quirúrgico. Métodos: Se trata de una investigación de campo, descriptiva de abordaje cualitativo. Resultados: Los clientes reportaron sentimientos de miedo y ansiedad, pero recibieron, en su mayoría, información del equipo de enfermería que fue escuchada y bienvenida durante la experiencia quirúrgica. Conclusión: El equipo de enfermería desarrolló una asistencia de calidad basada en principios de la humanización y de la asistencia integral, pero parece existir una necesidad de que estos profesionales se adecuen a aspectos socioculturales de los clientes.

Palabras clave: Enfermería Perioperatoria, Cuidados de Enfermería, Pacientes Internados.

${ }^{1}$ Faculdade Estácio de Alagoas (FAL), Maceió - AL. *E-mail: itamara28pa@hotmail.com

2Universidade Federal de Alagoas (UFAL), Maceió - AL. 


\section{INTRODUÇÃO}

O centro cirúrgico (CC) é um setor hospitalar que denota conduta imediata, seja para um procedimento terapêutico, de tratamento e correção, como também para análise diagnóstica. O indivíduo que necessita de intervenção cirúrgica comumente apresenta-se desconfortável pelo fato de colocar sua vida aos cuidados de uma equipe de saúde. Nos momentos que precedem o procedimento, ele se deparar com sentimentos que vão desde a esperança pela cura ao medo de uma inesperada e trágica condição que pode surgir durante ou após o procedimento, levando-os inclusive a morte (JORGETTO GV et al., 2017).

Esse setor é constituído por um conjunto de instalações em determinada área hospitalar nas quais permitem a efetuação de procedimentos cirúrgicos proporcionando condições de segurança e conforto para o paciente. Quanto aos aspectos físicos, possui circulação restrita, com acesso a Unidade de Terapia Intensiva (UTI), cujas suas finalidades visam a realização de procedimentos cirúrgicos, com retorno iminente dos pacientes às suas unidades de origem em condições melhores daquelas as quais chegou. Cabe ressaltar, que também estão inclusas nesse serviço uma efetiva atuação dos profissionais, sendo a equipe de enfermagem cirúrgica a categoria que presta assistência ao paciente em todos os períodos da conduta operatória, objetivando a prevenção de complicações físicas e emocionais, promovendo uma reabilitação e recuperação completa do paciente (SILVA DC e ALVIM AT, 2010; JORGETTO GV, et al. 2017)

O momento cirúrgico compreende o chamando perioperatório, que é entendido como o período que se inicia a indicação do procedimento cirúrgico e vai até o momento em que o paciente recebe a alta hospitalar, compreendendo ainda ao retorno do paciente as suas atividades normais. Nesse contexto, o perioperatório compreende três fases principais: pré-operatório, transoperatório e pós-operatório (MADEIRA MZA e OLIVEIRA EFV, 2011). A fase pré-operatória engloba a avaliação inicial e diagnóstica na qual são analisados os riscos de complicações em relação ao estado de saúde do paciente. Podem incluir a consulta, o jejum, o uso de medicações pré-anestésicas, o preparo intestinal, tricotomia, ou seja, é o período em que antecede a cirurgia. Subdivide-se em duas fases: o pré-operatório imediato que ocorre em até 24 horas antes do procedimento cirúrgico e o pré-operatório mediato que antecede o imediato, ou seja, todos os dias antes das 24 horas que antecedem a experiência cirúrgica (MADEIRA MZA e OLIVEIRA EFV, 2011).

A fase transoperatória consiste na intervenção realizada dentro do centro cirúrgico, estando nela compreendida a fase intraoperatória que vai desde o procedimento anestésico até a saída do cliente da sala de cirurgia. Nesse período, a equipe de enfermagem é responsável pelo preparo, estabelecendo e desenvolvendo as ações necessárias aos cuidados, de acordo com a especificidade da cirurgia e dentro do objeto da profissão. Existe necessidade de assistência individualizada e sistematizada, com planejamento em consonância com as necessidades identificadas na fase pré-operatória (MADEIRA MZA e OLIVEIRA EFV, 2011; MIRANDA AB et al., 2016).

Já o pós-operatório compreende a saída do paciente da sala cirúrgica até o retorno às suas atividades diárias. Esse período engloba três fases: o pós-operatório imediato essa fase inicia quando termina a cirurgia e tem duração de 24 horas; o pós-operatório mediato começando 24 horas após o encerramento da cirurgia, podendo se estender por 7 dias; e por último, o pós-operatório tardio que começa após o reconhecimento da alta (MADEIRA MZA e OLIVEIRA EFV, 2011).

Em todas essas fases os profissionais de enfermagem tornam-se importantes na condução da terapêutica, sendo elo e alicerce para o momento do estresse cirúrgico. Constitui um dos componentes principais no contexto cirúrgico, sendo composta por profissionais com responsabilidades e competência técnica variada. O Enfermeiro, o técnico de enfermagem e o auxiliar de enfermagem variam suas responsabilidades técnicas e operacionais de acordo com a gravidade e complexidade da cirurgia e de aspectos individuais dos sujeitos (SILVA DC e ALVIM NAT, 2010).

O enfermeiro é responsável por planejar as ações de enfermagem desenvolvidas durante o ato cirúrgico, além do gerenciamento de materiais e equipamentos. O técnico de enfermagem é o auxiliar direto do enfermeiro, com atribuições relativas à verificação do funcionamento, conservação e manutenção dos equipamentos fundamentais ao funcionamento preciso do centro cirúrgico; também é responsável pelo

REAS/EJCH | Vol.Sup.26 | e860 | DOI: https://doi.org/10.25248/reas.e860.2019 Página 2 de 8 
controle do material esterilizado, bem como o encaminhamento das peças cirúrgicas aos laboratórios especializados (SILVA DC e ALVIM NAT, 2010).

O circulante de sala (posição designada ao técnico de enfermagem) tem como atribuições, o atendimento direto das solicitações da equipe médica durante a cirurgia; além da manutenção da posição adequada do paciente; e controle de todos os equipamentos necessários. O auxiliar de enfermagem poderá ser responsável por montar a sala cirúrgica, além de desmontá-la e mantê-la organizada após o ato operatório (SILVA DC e ALVIM NAT, 2010). Além das funções técnicas atribuídas à equipe de enfermagem deverão estar inclusas também atividades educacionais e apoio psíquico como: a orientação aos pacientes e familiares; o preparo físico e emocional destes, além da avaliação contínua e o encaminhamento com vigilância e segurança ao centro cirúrgico (JOUCLAS VMG, et al., 2012).

Concerne-se o referimento aqui que um fator importante para o procedimento cirúrgico é a condição do paciente. As dificuldades enfrentadas para chegar à intervenção cirúrgica, os medos relativos ao procedimento, as comorbidades e aspectos pessoais e socioeconômicos são fatores que podem interferir no sucesso do procedimento, bem como na rapidez da recuperação (MATOS FGOA, et al., 2003).

Dessa forma, constata-se que no cotidiano dos centros cirúrgicos e durante as intervenções cirúrgicas é possível perceber alterações significativas, geradas exatamente, pela experiência cirúrgica, tendo influência direta com desequilíbrios físicos, emocionais e psicológicos, que podem dificultar a fase pós-operatória. Nesse sentido, a conduta de um olhar mais humano e holístico, voltado à singularidade dos sujeitos deve prevalecer (JUAN K, 2007).

Condutas quanto a percepção das necessidades emocionais e psicológicas do cliente durante o processo de intervenções cirúrgicas poderão gerar uma relação positiva entre os sujeitos do cuidar e a equipe de enfermagem, favorecendo uma percepção favorável do momento cirúrgico e da atuação desta equipe com resultados positivos na qualidade de vida e efetivação da saúde do paciente (MATOS FGOA et al., 2003).

Afirma-se, desta maneira, que os profissionais de saúde necessitam conhecer e atuar durante todas as fases do atendimento cirúrgico garantindo apoio e segurança ao paciente, relacionando os aspectos técnicos e científicos à condição individual e subjetiva dos sujeitos, para que a partir de então haja maior qualidade na assistência durante o momento operatório. Assim, diante da necessidade de perceber as sensações que os pacientes que vivenciaram experiências cirúrgicas, a partir das relações com a equipe de enfermagem cirúrgica, surgiu o seguinte questionamento: qual a percepção do cliente do pré-operatório imediato e transoperatório sobre o cuidado de enfermagem no centro cirúrgico? Para responder tal questionamento, o estudo teve como objetivo geral: analisar a percepção do cliente pré-operatório imediato e transoperatório sobre o cuidado de enfermagem no centro cirúrgico.

O bem-estar do cliente cirúrgico deve se constituir no principal objetivo dos profissionais que 0 assistem, uma vez que, nesse período alto nível de estresse pode acometer o paciente, gerando sentimentos que podem atingir negativamente em seu estado emocional. Dessa forma, esse momento pode tornar os clientes vulneráveis e dependentes (MATOS FGOA, et al., 2003). Assim, essa pesquisa justificou-se, pois através da avaliação da percepção dos indivíduos durante alguns períodos cirúrgicos, em específico o pré-operatório imediato e transoperatório, o indivíduo pôde apontar, em sua experiência cirúrgica, seu olhar sobre o cuidar desenvolvido pela equipe de enfermagem cirúrgica.

A compreensão da assistência prestada a partir da análise dos discursos daqueles que de fato são o foco da atenção pode refletir no entendimento da qualidade efetiva dos objetivos e metas da equipe deste setor, promovendo então uma efetivação e manutenção desta conduta.

\section{MÉTODOS}

Trata-se de um estudo de campo, descritivo de abordagem qualitativa, realizado em um hospital público, localizado na cidade de União dos Palmares, no estado de Alagoas. Esta unidade de saúde é o local de referência nesta cidade, possuindo um centro cirúrgico com duas salas. 
Esta pesquisa foi construída por procedimentos metodológicos baseados na coleta de dados através de uma entrevista com roteiro semiestruturado e para subsídios teóricos foi realizada revisão de literatura sobre a temática. Como critérios de inclusão para a pesquisa optou-se por clientes do hospital submetidos a processos cirúrgicos, tendo vivenciado os períodos pré-operatório imediato e transoperatório, e ainda, que já deveriam estar no período de recuperação de seu estado de hipnose e amnésia, estando conscientes de suas faculdades mentais. Foram totalizados 16 participantes entrevistados e para manter seu anonimato foram identificados como P1, P2, P3... P16, sendo interrompidas as entrevistas pela saturação dos dados.

Após a aprovação do Comitê de Ética e Pesquisa com número do parecer: 2.297.730 e CAAE: 72605717.0.0000.5012. Os sujeitos foram convidados a participar da pesquisa, sendo feita uma leitura do Termo de Consentimento Livre e Esclarecido (TCLE), junto ao pesquisador. Os pacientes que se aceitaram a participação assinaram o TCLE em duas vias, ficando uma em sua posse. Logo após, foram recrutados para um local reservado, fechado, com proteção de sua identidade. Cada entrevista teve uma duração média entre 20 e 30 minutos, onde foi utilizado um gravador para registro das falas que foram transcritas para a análise dentro dos objetivos desta pesquisa.

\section{RESULTADOS}

Ao total participaram desta pesquisa 16 pacientes, tendo aspectos referentes ao quesito sóciodemográfico e ao procedimento cirúrgico exposta na tabela abaixo (tabela 1).

Tabela 1 - Caracterização dos sujeitos da pesquisa, n= 16. União dos Palmares - AL, Brasil, 2017.

\begin{tabular}{|c|c|c|c|}
\hline Sexo & Faixa Etária & Tipos de Cirurgia & Escolaridade \\
\hline \multirow[t]{4}{*}{ Feminino: 15 participantes } & \multirow[t]{4}{*}{21 e 61 anos } & Cesarianas eletivas & \multirow{2}{*}{$\begin{array}{lr}\text { Ensino } & \text { Fundamental } \\
\text { completo: } & 10 \text { participantes }\end{array}$} \\
\hline & & Hemorroidectomia & \\
\hline & & Colpoperineoplastia & \multirow{2}{*}{$\begin{array}{l}\text { Ensino Médio Completo: } 5 \\
\text { participantes }\end{array}$} \\
\hline & & Histerectomias & \\
\hline Masculino: 1 participante & 66 anos & $\begin{array}{l}\text { Exérese de Cisto Dermóide } \\
\text { Herniorrafia Inguinal }\end{array}$ & $\begin{array}{l}\text { Não Alfabetizado: } \\
\text { participantes }\end{array}$ \\
\hline
\end{tabular}

Fonte: Dados da pesquisa, 2017.

Nas falas, foi possível perceber sentimentos positivos e negativos vivenciados pelos pacientes, antes e durante a experiência cirúrgica. Quanto aos negativos, sentimentos de ansiedade, medo e vergonha foram revelados, principalmente no momento anterior a cirurgia (pré-operatório). Assim, puderam-se destacar as seguintes falas:

\footnotetext{
"Entrei na sala de cirurgia tranquila, mas tive um pouco de medo quando cheguei" (P1).

"Só senti vergonha por causa do tipo da cirurgia" (P6).

"Estava ansiosa, mas não por medo, foi somente uma ansiedade normal, nada que pudesse prejudicar" (P8).
}

Mesmo diante de tais sensações os entrevistados relataram sentimentos de conforto ao vivenciarem um bom diálogo e uma informação acessível oferecida pela equipe de enfermagem. Isso pôde ser constatado nas seguintes falas:

"Sim, eles iam fazendo e explicando. Entendi, teve a hora de botar o soro, a moça lá falou, de limpar as costas, os fios que botaram, era pra ver o coração, outro a pressão e tinha aquele negócio do dedo, explicaram tudo, e o resto das coisas também" (P1). 
"[...] Todas às vezes, fui bem informada do que iam fazer. Sempre tive muita confiança no pessoal daqui" (P6).

Emergiram das falas aspectos relativos ao cuidado e ao apoio psicológico prestado pela equipe de enfermagem, a partir de um acolhimento e da criação de um vínculo. A satisfação quanto esta questão foi notória nas falas:

"Fui sim, o pessoal vinha, falava, fez a gente rir, eu e a outra menina que ia fazer curetagem. E na cirurgia perguntavam se estava tudo bem. Isso me fez se sentir confortável antes e na hora de fazer a cirurgia" (P1).

"Todos brincavam e relaxavam a pessoa, fui confortada em todos os momentos" (P8).

Além de conforto com diálogo e escuta, a enfermagem foi capaz de transmitir uma condição de tranquilidade, a partir de um cuidar físico baseado na aproximação física e no toque terapêutico que gerou uma sensação de estima por parte do profissional ao cliente.

"Teve uma enfermeira que segurou a minha mão na hora de ir e isso me deixou confiante" (P8).

Como reflexo de atitudes positivas que colaboram para o bem-estar dos sujeitos no momento cirúrgico, configurou-se uma relação de satisfação desde o primeiro momento ao resultado da cirurgia. Discursos positivos prevaleceram diante da assistência prestada pela equipe do cenário em questão, sendo notórios sentimentos de gratidão pela realização do procedimento cirúrgico, seja pelas vezes em que foram realizadas as cirurgias, ou pelo desejo de fazê-la, encontrando resolubilidade no hospital.

\section{"[...] estou satisfeita" (P1).}

"[...] já me operei sete vezes com essa, cinco aqui, todas foram uma maravilha meu filho. Não sei o que seria daqui sem esse hospital, nós somos abençoados por ter vocês cuidando do povo" (P6).

Contudo, aspectos referentes às informações sobre as intervenções no pré-operatório e transoperatório não se deu em uniformidade para todos os sujeitos, visto existirem falas que demonstraram pouca informação ou explicações fora da capacidade de assimilação dos indivíduos devido a aspectos socioculturais dos mesmos.

"Fui seu menino, eu não tava entendendo muita coisa não, porque tenho pouca instrução, e muita coisa eu não sabia o que era. Aí eu mandei a menina explicar melhor e eu entendi melhor. Entendi até onde deu!" (P16).

"Não, só foram fazendo, sem falar nada. Eu fiquei nervosa [...] Fiquei muito angustiada com esse descaso" (P4).

\section{DISCUSSÃO}

A internação hospitalar para o paciente significa o distanciamento de seu cotidiano para um ambiente com outras rotinas e normas rígidas. Neste sentido, vivenciar a hospitalização com a espera de uma intervenção cirúrgica pode levar o paciente a conflitos internos que envolvem sentimentos de medo e ansiedade (ANTONIO PS, et al., 2002). Corroborando, na atual pesquisa foram encontrados sentimentos como o medo, a vergonha e a ansiedade.

O medo do desconhecido é o principal motivo de insegurança e ansiedade do paciente cirúrgico. Esses sentimentos são amenizados com a experiência, mas podem aparecer em maior ou menor intensidade, mediante a complexidade cirúrgica. A internação hospitalar é vista como uma experiência desagradável por aquele que a vivencia (FIGHERA J e VEIRO EV, 2005; RAZERA APR e BRAGA EM, 2011). 
O qual pode surgir a partir da necessidade da utilização de recursos tecnológicos, que podem ser invasivos e dolorosos; uso de linguagem técnica capaz de aumentar a ansiedade do paciente em relação ao seu quadro patológico; pela inquietação de estar em um ambiente estranho ao mesmo em que, partilha o mesmo espaço físico com pessoas desconhecidas; e pela preocupação com a sua evolução clínica (RAZERA APR e BRAGA EM, 2011).

Outro aspecto apresentado no estudo a partir dos depoimentos são momentos de ansiedade em relação à violação interior do corpo, mesmo com a existência da necessidade da realização da cirurgia. É um fato que ocorre por um sentimento de agressão que o procedimento cirúrgico pode representar a integridade física do sujeito.

Quanto à nudez no ambiente cirúrgico, é certo não se banalizar o corpo nu, mesmo que isso aconteça de forma costumeira, pois cada exposição do corpo nu em pacientes diferentes expõe a subjetividade de cada indivíduo e a nudez traz consigo um espaço pessoal dentro de suas particularidades. Ao desnudar, o profissional invade esse espaço e, isto implícita tabus que se encontram na base da vida social (SANTOS RM, et al., 2010).

A equipe de enfermagem tende a estar em contato direto com o paciente durante todo o perioperatório, necessitando estar atenta aos sofrimentos e aflições dos sujeitos, seja ela revelada a partir da comunicação verbal ou gestual (JUAN K, 2007).

O diálogo e as explicações referentes à conduta cirúrgica deverão estar presentes na rotina destes profissionais, e a comunicação neste setor deverá ser contínua, na busca de compreender as reais necessidades do cliente (JUAN K, 2007). Assim, através das falas percebe-se a troca de informações para manter o indivíduo ciente sobre a sua situação e sobre o ambiente que o rodeia.

Nesse contexto, entende-se ainda, que conhecer a percepção do sujeito sobre os cuidados recebidos, como também os fatores que podem implicar na sua satisfação ou insatisfação são de fundamental importância. O paciente satisfeito e bem informado colabora com a assistência prestada refletindo no sucesso do tratamento (VAITSMAN J e ANDRADE GRB, 2005).

A satisfação do paciente pode ser uma definição de uma resposta afetiva e de avaliação dos serviços de saúde prestado, é constitui uma ferramenta contributiva para uma reflexão que pode resultar em melhorias contínuas do trabalho em equipe (DIAS AB, et al., 2008).

Ainda quanto à satisfação relativa ao cuidado de enfermagem, o contato físico também poderá transmitir o conforto necessário para o paciente cirúrgico. O toque terapêutico é capaz de proporcionar conforto e calor humano, transmitindo uma mensagem para o paciente de que ele não está só diante de toda a situação vivenciada (DIAS AB, et al., 2008).

$O$ ato de tocar possui relação íntima com o exercício profissional, uma vez que é executado o exame físico, ferramenta essencial do processo de enfermagem. O toque aproxima o profissional do paciente e dos objetivos da enfermagem. Assim, o toque terapêutico está inserido, no contexto de ações não verbais, possibilitando que a enfermagem possa demonstrar suas habilidades técnicas, como também, sua capacidade de ser compreensiva e solidária (DIAS AB, et al., 2008). Neste estudo, há que se destacar ainda, que pacientes quando chegam ao tratamento na alta complexidade, em específico no centro cirúrgico, esbarram em protocolos e por uma longa fila de espera. Neste estudo, foi possível observar sentimentos de satisfação em falas pela resolubilidade do hospital em questão, pela facilidade de acesso ao procedimento cirúrgico, onde os indivíduos expressam sentimentos de gratidão pela realização do procedimento cirúrgico.

Quando a este quesito, estudos apontam para discursos que remetam a gentilezas e favores, no subjetivo da cordialidade. Em instituições de saúde, tais situações podem suprimir as reais sensações e vivências quanto aos pontos negativos de qualquer assistência, pois o desejo maior de ter conseguido a cirurgia, sobrepõe-se as demais situações vivenciadas, dentre elas uma má assistência da equipe (ROHRBACHE I e HADDAD L, 2017). Indivíduos que se encontram sobre cuidados cirúrgicos necessitam de um olhar humanizado, que percorre aspectos tanto físicos, como mentais e espirituais. Olhar integralmente os sujeitos 
é perceber aspectos referentes, inclusive ao contexto sociocultural, configurando a comunicação em saúde um aspecto singular entre o profissional e o usuário. Muitas vezes, a dificuldade de entendimento do conteúdo científico expresso pela equipe de enfermagem compromete a vivência cirúrgica (RAZERA APR e BRAGA EM, 2011).

A fácil comunicação é uma das formas de realizar, ou mesmo, de melhorar os cuidados de enfermagem. É por meio da comunicação aberta e real que se desenvolve a aproximação entre sujeitos, sendo uma forma de estreitar os laços, numa relação de envolve a integração de culturas com o objetivo de favorecer maior entendimento e compreensão de experiências (JUAN K, 2007).

Uma experiência cirúrgica de qualidade é reflexo da execução do conhecimento técnico-científico e de uma prática humanizada, desenvolvida pela equipe de saúde do bloco cirúrgico. A equipe multiprofissional tem o dever de resgatar valores humanos no seu processo de trabalho, de forma a desconstruir o cuidado mecanizado e feito rotineiramente. Assim, a equipe pode proporcionar uma práxis transformadora da relação entre profissional e paciente, promovendo um atendimento holístico (NASCIMENTO KTS, et al., 2014).

Neste sentido, favorecer uma assistência humanizada e individualizada é um elemento balizador de práticas assistenciais de qualidade e tem na comunicação com o paciente, a partir sua apresentação verbal e gestual, uma ferramenta indispensável para a execução do cuidado real.

A Enfermagem que humaniza, a partir dos princípios da escuta ativa e do diálogo, é capaz de favorecer bons sentimentos, mesmo a aqueles em situações extremas por meio de ferramentas e estratégias como a Sistematização da Assistência de Enfermagem Perioperatória (SAEP) a qual visa através de linha de organização e planejamento prevenir complicações resultantes do processo cirúrgico.

A SAEP tem como base a construção de um plano da assistência perioperatória durante todas as fases da experiência cirúrgica, oferecendo apoio emocional ao paciente/família, como também ajudando-os na compreensão dos problemas de saúde do mesmo (BROCA PV e FERREIRA MA, 2015; FONSECA e PENICHE, 2009).

\section{CONCLUSÃO}

Esse estudo buscou refletir sobre os cuidados de enfermagem no período pré-operatório imediato e transoperatório na visão do paciente. Diante dos resultados, constatou-se que a assistência de Enfermagem prestada no cenário da pesquisa, revelou-se como de qualidade visto a satisfação dos pacientes expressa nas falas. Além disso, pôde-se perceber um olhar holístico na assistência prestada nos períodos cirúrgicos em que esse estudo voltou a sua atenção, com uma equipe de enfermagem focada em aspectos físicos, mentais e sociais dos clientes cirúrgicos.

Em destaque à inter-relação entre a equipe de enfermagem e pacientes, pode-se compreender que uma assistência de qualidade parece ter relação com uma postura que respeite o sujeito enquanto ser humano, com cordialidade, escuta e diálogo. Observou-se que cultivar a autonomia do paciente, pode ser um diferencial nas práticas que antecedem a cirurgia. Essa autonomia pôde ser percebida pela a acessibilidade às informações e pela comunicação ativa em todas as fases do período operatório, pois aumentou a percepção do paciente em sua capacidade de controlar a situação vivenciada. Pode-se perceber que ainda existem lacunas neste processo, visto alguns sujeitos não serem alcançados em sua plenitude pelo acolhimento e por atitudes que garantam uma relação de confiança. Parece existir uma necessidade dos profissionais de saúde se adequarem aos aspectos socioculturais dos clientes, sendo mais acessível a esta condição. Porém, tais pontos observados aqui poderão servir de alerta para que uma atitude integral e humana se complete nas singularidades dos sujeitos de maneira a universalizar o sentimento de satisfação para a experiência cirúrgica quanto aos cuidados da equipe de enfermagem. Vale ressaltar ainda que essa humanização deve transversalizar, não somente os centros cirúrgicos, mas todo o complexo que envolve o processo da cirurgia, fundamentando-se em conceitos nos quais os pacientes possam ser atendidos em sua integridade enquanto ser. 


\section{REFERÊNCIAS}

1. JORGETTO GV, et al. Assistência de enfermagem a pacientes cirúrgicos: avaliação comparativa. Revista Eletrônica de Enfermagem, 2005; 07(03): 273-277.

2. SILVA DC, ALVIM NAT. Ambiente do Centro Cirúrgico e os elementos que o integram: implicações para os cuidados de enfermagem. Revista Brasileira de Enfermagem, 2010; 63(3): 427-434.

3. MADEIRA MZA, OLIVEIRA EFV. A assistência de enfermagem perioperatória e a satisfação do paciente. Revista Interdisciplinar, 2011; 4(2): 9-15.

4. MIRANDA AB, et al. Posicionamento cirúrgico: cuidados de enfermagem no transoperatório. Revista SOBECC, 2016; 21(1): 52-58.

5. JOUCLAS VMG, et al. Qualidade do cuidado de enfermagem transoperatório e de recuperação anestésica de acordo com a satisfação do cliente. Revista Cogitare Enfermagem, 2012; 46(esp): 138-47.

6. MATOS FGOA, et al. Aspectos emocionais do paciente cirúrgico. In: Anais do 1ำ Seminário Nacional Estado e Políticas Sociais em Cascavel/PR, Brasil [internet], 2003.

7. JUAN K. O impacto da cirurgia e os aspectos psicológicos do paciente: uma revisão. Revista Psicologia Hospitalar, 2007; 5(1): 48-59.

8. BARDIN L. Análise de conteúdo. Ed. São Paulo; 2011.

9. ANTONIO PS, et al. Fatores geradores de sentimentos do paciente internado frente ao cancelamento de cirurgias. Revista Eletrônica de Enfermagem, 2002; 4(1): 33-39.

10. FIGHERA J, VIERO EV. Vivências do paciente com relação ao procedimento cirúrgico: fantasias e sentimentos mais presentes. Revista SBPH, 2005; 8(2): 51-63.

11. RAZERA APR, BRAGA EM. A importância da comunicação durante o período de recuperação pós-operatória. Revista Escola de Enfermagem USP, 2011; 45(3): 632-637.

12. SANTOS RM, et al. A enfermeira e a nudez do paciente. Revista Brasileira de Enfermagem, 2010; 63(6): 877-886.

13. VAITSMAN J, ANDRADE GRB. Satisfação e responsividade: formas de medir a qualidade e a humanização da assistência à saúde. Revista Ciência e Saúde Coletiva, 2005; 10(3): 599-613.

14. DIAS AB, et al. O toque afetivo na visão do enfermeiro. Revista Brasileira de Enfermagem, 2008; 61(5): 603-607.

15. ROHRBACHE I, HADDAD LH. Satisfação de pacientes após cirurgia refrativa de monovisão avançada. Revista Brasileira de Oftalmologia, 2017; 76(4): 190-193.

16. NASCIMENTO KTS, et al. Cuidar integral da equipe multiprofissional: discurso de mulheres em pré-operatório de mastectomia. Escola Anna Nery Revista de Enfermagem, 2014; 18(3): 66-79.

17. BROCA PV, FERREIRA MA. Processo de comunicação na equipe de enfermagem fundamentado no diálogo entre Berlo e King. Escola Anna Nery Revista de Enfermagem, 2015; 19(3): 36-45.

18. FONSECA RMP, PENICHE ACG. Enfermagem em centro cirúrgico: trinta anos após criação do Sistema de Assistência de Enfermagem Perioperatória. Revista Acta Paulista de Enfermagem, 2009; 22(4): 428-33. 